\title{
A note on the zeros of approximations of the Ramanujan E-function
}

\section{Andrés Chirre ${ }^{1} \cdot$ Oswaldo Velásquez Castañón ${ }^{2}$}

Received: 4 May 2020 / Accepted: 8 August 2020 / Published online: 28 August 2020

(c) The Author(s) 2020

\begin{abstract}
In this paper we review the study of the distribution of the zeros of certain approximations for the Ramanujan $\Xi$-function given by Ki (Ramanujan J 17(1):123-143, 2008), and we provide new proofs of his results. Our approach is motivated by the ideas of Velásquez (J Anal Math 110:67-127, 2010) in the study of the zeros of certain sums of entire functions with some condition of stability related to the Hermite-Biehler theorem.
\end{abstract}

Keywords Ramanujan zeta function - Riemann hypothesis - Zeros of approximations of the Ramanujan $\Xi$-function - Distribution of zeros of entire functions

Mathematics Subject Classification 11M26 - 30D10

A. C. was supported by Grant 275113 of the Research Council of Norway. O. V. was supported by MATH-AmSud program: project MZFTTA, Peruvian Grant Nos. 310-2014-FONDECYT and CG-176-2015-FONDECYT.

$凶$ Andrés Chirre

carlos.a.c.chavez@ntnu.no

Oswaldo Velásquez Castañón oswaldo@imca.edu.pe

1 Department of Mathematical Sciences, Norwegian University of Science and Technology, 7491 Trondheim, NO, Norway

2 Instituto de Matemática y Ciencias Afines, Universidad Nacional de Ingeniería, Lima, Peru 


\section{Introduction}

\subsection{Background}

Let $\tau(n)$ be the Ramanujan's tau-function, defined by

$$
\Delta(z)=\sum_{n=1}^{\infty} \tau(n) q^{n}=z \prod_{n=1}^{\infty}\left(1-q^{n}\right)^{24}
$$

where $q=e^{2 \pi i z}$, and $\operatorname{Im} z>0$. It is well known that $\Delta(z)$ spans the space of cusp forms of dimension -12 associated with the unimodular group. The associated Dirichlet series and Euler product for $\Delta(z)$ are given by

$$
L(s)=\sum_{n=1}^{\infty} \frac{\tau(n)}{n^{s}}=\prod_{p}\left(1-\tau(p) p^{-s}+p^{11-2 s}\right)^{-1},
$$

where the series and the product are absolutely convergent for $\operatorname{Re} s>13 / 2$.

Let us define the Ramanujan $\Xi$-function, denoted by $\Xi_{R}(s)$, as follows:

$$
\Xi_{R}(s)=(2 \pi)^{i s-6} L(-i s+6) \Gamma(-i s+6),
$$

where $\Gamma(s)$ is the Gamma function. Another representation for $\Xi_{R}(s)$ is given by

$$
\Xi_{R}(s)=\int_{-\infty}^{\infty} \phi(t) e^{i s t} \mathrm{~d} t
$$

where

$$
\phi(t)=e^{-2 \pi \cosh (t)} \prod_{k=1}^{\infty}\left[\left(1-e^{-2 \pi k e^{t}}\right)\left(1-e^{-2 \pi k e^{-t}}\right)\right]^{12} .
$$

In [1], Hardy highlighted the importance of the location of the zeros of $\Xi_{R}(s)$ in the strip $|\operatorname{Im}(s)| \leq \frac{1}{2}$. The Riemann hypothesis for the Ramanujan zeta function states that all zeros of $\Xi_{R}(s)$ are real.

\subsection{Zeros of the approximations $\Xi_{F}(s)$}

The purpose of this paper is to study the distribution of the zeros of certain approximations for the Ramanujan $\Xi$-function. Inspired in the representation (1.1), Ki [2] defined these approximations as follows: Let $F$ be a finite sequence of complex numbers $a_{0}, a_{1}, \ldots, a_{n}$ such that at least one of them is different from zero. We define the function

$$
\Xi_{F}(s)=\int_{-\infty}^{\infty} \phi_{F}(t) e^{i s t} \mathrm{~d} t
$$


where

$$
\phi_{F}(t)=e^{-2 \pi \cosh t}\left(\sum_{m=0}^{n} a_{m} e^{-2 \pi m e^{t}}\right)\left(\sum_{m=0}^{n} \overline{a_{m}} e^{-2 \pi m e^{-t}}\right) .
$$

We recall that $\overline{\Xi_{F}(\bar{s})}=\Xi_{F}(s)$, and one can see that for some sequences $F_{k}$, the function $\Xi_{F_{k}}(s)$ converges uniformly to $\Xi_{R}(s)$ on all compact subsets of $\mathbb{C}$.

Throughout this paper, we will study the distribution of the zeros of the function $C_{F}(s):=\Xi_{F}(-i s)$. Note that the zeros of $C_{F}(s)$ are symmetric respect to the line $\operatorname{Re} s=0$. Using the argument principle, Ki [2, Theorem 1] established for $T \geq 2$ that $^{1}$

$$
N\left(T, C_{F}\right)=\frac{T}{\pi} \log \frac{T}{e \pi}+O(\log T)
$$

where $N\left(T, C_{F}\right)$ stands for the number of zeros of $C_{F}(s)$ such that $1 \leq \operatorname{Im} s<T$, counting multiplicity. In the lower half-plane a similar result holds. Moreover, using the method developed by Levinson [3], he stated that

$$
\bar{N}\left(T, C_{F}\right)-\overline{N_{1}}\left(T, C_{F}\right)=O(T),
$$

where $\bar{N}\left(T, C_{F}\right)$ stands for the number of zeros of $C_{F}(s)$ such that $|\operatorname{Im} s|<T$, counting multiplicity and $\overline{N_{1}}\left(T, C_{F}\right)$ denotes the number of simple zeros such that $|\operatorname{Im} s|<T$ and $\operatorname{Re} s=0$. In a sense, it means that almost all zeros of $C_{F}(s)$ lie on the line $\operatorname{Re} s=0$ and are simple. Our first goal is to establish a refinement of (1.2).

Theorem 1 For $T \geq 2$ we have

$$
0 \leq \bar{N}\left(T, C_{F}\right)-\overline{N_{1}}\left(T, C_{F}\right) \leq\left(32 n+\frac{32 \ln (2 n+1)}{\pi}\right) T+O(1) .
$$

On the other hand, $\mathrm{Ki}$ [2, Theorem 2] found a result about the vertical distribution of the zeros of $C_{F}(s)$, based on the zeros of the function $\psi_{F}(s)$, defined by

$$
\psi_{F}(s)=\pi^{-s} \sum_{m=0}^{n} a_{m}(2 m+1)^{-s}
$$

Let $k \geq 0$ be an integer such that $P(1)=P^{\prime}(1)=\cdots=P^{(k-1)}(1)=0$ and $P^{(k)}(1) \neq 0$, where $P(y)=\sum_{m=0}^{n} a_{m} y^{m}$.

Theorem 2 Let $\Delta_{*}<\Delta_{* *}$ be positive real numbers. Suppose that $\psi_{F}(s-k)$ has finitely many zeros in $-\Delta_{* *}<\operatorname{Re} s<\Delta_{*}$. Let $\delta$ be such that $0<\delta<\Delta_{*}$. Then all but finitely many zeros of $C_{F}(s)$ which lie in $|\operatorname{Re} s| \leq \delta$ are on the line $\operatorname{Re} s=0$. In particular, all but finitely many zeros of $C_{F}(s)$ are on the line $\operatorname{Re} s=0$, if $\psi_{F}(s-k)$ has finitely many zeros in $\operatorname{Re} s>-\Delta_{* *}$.

\footnotetext{
1 Throughout the paper we use the Vinogradov's notation $f=O(g)$ (or $f \ll g$ ) to mean that $|f(t)| \leq$ $C|g(t)|$ for a certain constant $C>0$ and $t$ sufficiently large.
} 
$\mathrm{Ki}$ included a second proof for the second part of Theorem 2. In particular, this second proof gave information about the simplicity of the zeros of $C_{F}(s)$. Anyway, Ki conjectured that second case for $\psi_{F}(s-k)$ is not possible. On the other hand, using (2.7) it is clear that $\psi_{F}(s-k)$ has the same set of zeros of a Dirichlet polynomial in the framework of [4, Sect. 12.5]. The set of zeros of a Dirichlet polynomial is quasi-periodic (see [5, Appendix 6, p. 449]). Then, if $s_{0}=\sigma_{0}+i \tau_{0}$ is a zero of the Dirichlet polynomial, for any $\varepsilon>0$ we can construct a sequence $\left\{s_{n}=\sigma_{n}+i \tau_{n}\right\}_{n \in \mathbb{N}}$ of zeros, such that $\left.\sigma_{n} \in\right] \sigma_{0}-\varepsilon, \sigma_{0}+\varepsilon$ [ for all $n \in \mathbb{N}$ and $\tau_{n} \rightarrow \pm \infty$. This implies that each open vertical strip has no zeros or has infinite zeros. Therefore, the hypothesis in Theorem 2 is reduced to $\psi_{F}(s-k)$ having no zeros in $-\Delta_{* *}<\operatorname{Re} s<\Delta_{*}$. Our second goal in this paper is to give a new proof of this result.

Theorem 3 Let $\Delta_{*}<\Delta_{* *}$ be positive real numbers. Suppose that $\psi_{F}(s-k)$ has no zeros in $-\Delta_{* *}<\operatorname{Re} s<\Delta_{*}$. Let $\delta$ be such that $0<\delta<\Delta_{*}$. Then all but finitely many zeros of $C_{F}(s)$ which lie in $|\operatorname{Re} s| \leq \delta$ are on the line $\operatorname{Re} s=0$ and are simple.

We highlight that our proof includes information about the simplicity of the zeros for the first case. The key relation between the functions $C_{F}(s)$ and $\psi_{F}(s-k)$ is due to de Bruijn [6, p. 225], who showed that

$$
C_{F}(s)=\sum_{m=k}^{\infty} b_{m} \psi_{F}(s-m) \Gamma(s-m)+\sum_{m=k}^{\infty} \frac{}{b_{m} \psi_{F}(-s-m)} \Gamma(-s-m),
$$

where $b_{m}$ are complex numbers and $b_{k} \neq 0$.

\subsection{Strategy outline}

Our approach is motivated by a result of Velásquez [7, Theorem 36], about the distribution of the zeros of a function of the form $f(s)=h(s)+h^{*}(2 a-s)$, where $h(s)$ is a meromorphic function ${ }^{2}$, and $a \in \mathbb{R}$. This result can be regarded as a generalization of the necessary condition of stability for the function $h(s)$, in the Hermite-Biehler theorem [5, 21, Part III, Lecture 27]. In our case, using an auxiliary function $W_{F}(s)$, we have the representation $C_{F}(s)=h(s)+h^{*}(-s)$, where $h(s)=W_{F}(-i s-i / 2)$. Some estimates of $h(s)$ due to Ki [2, Theorem 2.1] play an important role to establish the necessary growth conditions in [7, Theorem 36]. On the other hand, the strong relation between the zeros of $h(s)$ and $\psi_{F}(\mathrm{~s}$ ) (see (2.5)) implies that one study the distribution of zeros of $\psi_{F}(\mathrm{~s})$, as a set of zeros of a Dirichlet polynomial.

Throughout the paper, we fix a sequence $F$. For a function $f(s)$ and the parameters $\sigma_{1}<\sigma_{2}$, and $T_{1}<T_{2}$, we denote the counting function

$$
\begin{aligned}
& N\left(\sigma_{0}, \sigma_{1}, T_{1}, T_{2}, f\right)=\#\left\{s \in \mathbb{C}: f(s)=0, \sigma_{0}<\sigma<\sigma_{1}, T_{1}<\tau<T_{2}\right\}, \\
& \widehat{N}\left(\sigma_{0}, \sigma_{1}, T_{1}, T_{2}, f\right)=\#\left\{s \in \mathbb{C}: f(s)=0, \sigma_{0} \leq \sigma \leq \sigma_{1}, T_{1}<\tau<T_{2}\right\},
\end{aligned}
$$

$\overline{2 \text { For a meromorphic function }} h(s)$, we define the function $h^{*}(s)=\overline{h(\bar{s})}$. 
where, in both cases, the counts are with multiplicity, and

$$
N_{0}^{\prime}(T, g)=\#\{s \in \mathbb{C}: g(s)=0, \operatorname{Re} s=0,|\operatorname{Im} s|<T\}
$$

where the count is without multiplicity.

\section{Preliminary results}

In this section we collect several results for our proof. We highlight that in [2, Proposition 2.3], Ki showed that there is a constant $\beta_{0}>0$ such that $C_{F}(s) \neq 0$, for $|\operatorname{Re} s| \geq \beta_{0}$. This implies that for $\beta \geq \beta_{0}$,

$$
\bar{N}\left(T, C_{F}\right)=N\left(-\beta, \beta,-T, T, C_{F}\right)
$$

Therefore, we can restrict our analysis of the zeros in vertical strips. Now, let us start to find a new representation for $C_{F}(s)$. We define the entire function

$$
W_{F}(s)=\int_{-\infty}^{\infty} \tilde{\phi}_{F}(t) e^{i s t} \mathrm{~d} t
$$

where

$$
\tilde{\phi}_{F}(t)=\frac{e^{-2 \pi \cosh t}}{e^{t / 2}+e^{-t / 2}}\left(\sum_{m=0}^{n} a_{m} e^{-2 \pi m e^{t}}\right)\left(\sum_{m=0}^{n} \overline{a_{m}} e^{-2 \pi m e^{-t}}\right) .
$$

Then, we obtain the following relation:

$$
C_{F}(s)=W_{F}\left(-i s-\frac{i}{2}\right)+W_{F}\left(-i s+\frac{i}{2}\right)
$$

If we denote by

$$
h(s)=W_{F}\left(-i s-\frac{i}{2}\right)
$$

we rewrite (2.2) as

$$
C_{F}(s)=h(s)+h^{*}(-s) .
$$

This representation allows us to use the following result (see [7, Theorem 36]).

Theorem 4 Let $\sigma_{0}>0$ be a parameter and $h(s)$ be an entire function such that $h(s) \neq 0$ for $\operatorname{Re} s=\sigma_{0}$. We define the entire function

$$
f(s)=h(s)+h^{*}(-s) .
$$


Suppose that the function

$$
F(s)=\frac{h^{*}(-s)}{h(s)}
$$

satisfies the following conditions.

(i) $F(s) \neq \pm 1$ on the line $\operatorname{Re} s=\sigma_{0}$, and for some $\tau_{0}>0$ we have $|F(s)|<1$ for $s=\sigma_{0}+i \tau$ with $|\tau| \geq \tau_{0}$.

(ii) There exist an increasing function $\varphi: \mathbb{R} \rightarrow \mathbb{R}$, a constant $K>0$ and sequences $\left\{T_{m}\right\}_{m \in \mathbb{N}},\left\{T_{m}^{*}\right\}_{m \in \mathbb{N}}$ such that $\lim _{m \rightarrow \infty} T_{m}=\lim _{m \rightarrow \infty} T_{m}^{*}=\infty$,

$$
T_{m} \leq T_{m+1} \leq \varphi\left(T_{m}\right), \quad T_{m}^{*} \leq T_{m+1}^{*} \leq \varphi\left(T_{m}^{*}\right) \quad \text { for } m \in \mathbb{N}
$$

and $|F(s)|<e^{K|s|}$, for $s=\sigma+i \tau$ with $0 \leq \sigma \leq \sigma_{0}$ and $\tau=T_{m}, \tau=-T_{m}^{*}$, for $m \in \mathbb{N}$.

Then, for $T \geq 2$, we have that

$$
N\left(-\sigma_{0}, \sigma_{0},-T, T, f\right)-N_{0}^{\prime}(T, f) \leq 4 \widehat{N}\left(0, \sigma_{0},-\varphi(2 T), \varphi(2 T), h\right)+O(1),
$$

To prove that the function $h(s)$ defined in (2.3) satisfies the conditions of the previous theorem, we will use the estimates used by Ki. By [2, Eq. (2.1)], using the change of variable $s \mapsto-i s-i / 2$, we have that

$$
h(s)=\Gamma(s-k)\left(b_{k} \psi_{F, k}(s)+O\left(|s|^{-1 / 2}\right)\right)
$$

holds uniformly on the half-plane $\operatorname{Re} s \geq-1 / 4$ and $|s|$ sufficiently large. On the other hand, by [2, Theorem 2.1] it follows using the change of variable $s \mapsto-i s+i / 2$ : for $\Delta>0$ sufficiently large,

$$
\frac{h^{*}(-s)}{\Gamma(s-k-1)|\tau|^{\mu(\sigma)}}=O(1)
$$

for $s=\sigma+i \tau$ with $0 \leq \sigma \leq \Delta$ and $|\tau| \geq 1$, and the function $\mu(\sigma)$ is given by

$$
\mu(\sigma)=\left\{\begin{array}{lll}
1-\sigma, & \text { si } & 0 \leq \sigma \leq 1 \\
0, & \text { si } & \sigma>1
\end{array}\right.
$$

Finally, we will need to establish bounds for the right-hand side of (2.4) to estimate the number of zeros of $h(s)$. The relation (2.5) tells us that we must study the behavior of the zeros of $\psi_{F}(s)$. We define $\psi_{F, k}(s):=\psi_{F}(s-k)$. Thus, using (1.3) this function can be written as

$$
\psi_{F, k}(s)=\sum_{m=0}^{n} a_{m} e^{-\ln ((2 m+1) \pi)(s-k)}=e^{-\ln ((2 n+1) \pi)(s-k)}\left[\sum_{m=0}^{n} p_{m} e^{\beta_{m} s}\right],
$$


where $p_{m}=\left(a_{n-m}\right) e^{-\beta_{m} k}$ and $\beta_{m}=\ln ((2 n+1) /(2(n-m)+1))$, for $0 \leq m \leq n$. The sum on the right-hand side of (2.7) is a Dirichlet polynomial in the framework [4, Sect. 12.5].

Proposition 5 Let $Z\left(\psi_{F, k}\right)$ denote the set of zeros of $\psi_{F, k}(s)$.

(1) There is a positive real number $c_{0}$ such that $Z\left(\psi_{F, k}\right) \subset\left\{s \in \mathbb{C}:|\operatorname{Re} s|<c_{0}\right\}$.

(2) For $T_{1}<T_{2}$ and $c \geq c_{0}$, we have that

$$
N\left(-c, c, T_{1}, T_{2}, \psi_{F, k}\right) \leq n+\frac{\ln (2 n+1)}{2 \pi}\left(T_{2}-T_{1}\right) .
$$

(3) Let $K \subset \mathbb{C}$ such that $|\operatorname{Re} s| \leq M$ for $s \in K$, and some $M>0$. Suppose that $K$ is uniformly bounded from the zeros of $\psi_{F, k}(s)$, i.e.,

$$
\inf \left\{|s-z|: s \in K, z \in Z\left(\psi_{F, k}\right)\right\}>0 \text {. }
$$

Then, $\inf \left\{\left|\psi_{F, k}(s)\right|: s \in K\right\}>0$.

Proof See [4, Theorems 12.4, 12.5 and 12.6].

\section{Proofs of Theorems 1 and 3}

\subsection{Proof of Theorem 1}

Let us define the function

$$
F(s)=\frac{h^{*}(-s)}{h(s)}
$$

Since that $h(s)$ and $h^{*}(-s)$ are entire functions, we can choose $\sigma_{0}>0$ sufficiently large such that $F(s) \neq \pm 1$ and $h(s) \neq 0$ on the line $\operatorname{Re} s=\sigma_{0}$. Using (2.5) and (2.6) we get for $s=\sigma+i \tau$ with $0 \leq \sigma \leq \sigma_{0}$ and $|\tau|$ sufficiently large,

$$
\begin{aligned}
& F(s) \\
& \quad=\frac{O(1) \Gamma(s-k-1)|\tau|^{\mu(\sigma)}}{\Gamma(s-k)\left(b_{k} \psi_{F, k}(s)+O\left(|s|^{-1 / 2}\right)\right)} \\
& \quad=\frac{O(1)|\tau|^{\mu(\sigma)}}{(s-k-1)\left(b_{k} \psi_{F, k}(s)+O\left(|s|^{-1 / 2}\right)\right)} .
\end{aligned}
$$

Now, we analyze the behavior of $F(s)$ on the line $\operatorname{Re} s=\sigma_{0}$. Note that $\mu\left(\sigma_{0}\right)=0$. On another hand, the line $\operatorname{Re} s=\sigma_{0}$ is uniformly bounded from the zeros of $\psi_{F, k}(s)$. Then, recalling that $b_{k} \neq 0$, by Proposition 5 and the triangle inequality we get

$$
\left|b_{k} \psi_{F, k}(s)+O\left(|s|^{-1 / 2}\right)\right| \gg 1,
$$


for $s=\sigma_{0}+i \tau$, with $|\tau|$ sufficiently large. Inserting this in (3.2), it follows

$$
|F(s)| \ll \frac{1}{|s-k-1|} .
$$

Therefore, for $s=\sigma_{0}+i \tau$ with $|\tau|$ sufficiently large we conclude that $|F(s)|<1$. This implies (i) of Theorem 4. Let us prove (ii) of Theorem 4. For each $m \in \mathbb{Z}$ we consider the rectangle

$$
R_{m}=\left\{s \in \mathbb{C}:-\sigma_{0}<\operatorname{Re} s<\sigma_{0}, m<\operatorname{Im} s<m+1\right\} .
$$

We divide this rectangle into $2 n+1$ subrectangles $R_{m, j}$ defined by

$$
R_{m, j}=\left\{s \in \mathbb{C}:-\sigma_{0}<\operatorname{Re} s<\sigma_{0}, \quad m+\frac{j-1}{2 n+1}<\operatorname{Im} s<m+\frac{j}{2 n+1}\right\},
$$

for $j \in\{1,2, \ldots, 2 n+1\}$. By Proposition 5 we have that $N\left(-\sigma_{0}, \sigma_{0}, m, m+\right.$ $\left.1, \psi_{F, k}\right) \leq 2 n$. So, there exists $j_{0}$ such that $\psi_{F, k}(s)$ does not vanish in $R_{m, j_{0}}$. Let us write

$$
T_{m}=m+\frac{j_{0}-\frac{1}{2}}{2 n+1}
$$

Note that $m<T_{m}<m+1$. Then, if we define $\varphi(x)=x+2$, we have that

$$
m<T_{m}<m+1<T_{m+1}<m+2<T_{m}+2=\varphi\left(T_{m}\right) .
$$

Let $K=\left\{s \in \mathbb{C}:-\sigma_{0}<\operatorname{Re} s<\sigma_{0}, \quad \operatorname{Im} s=T_{m}, m \in \mathbb{Z}\right\}$. For any $s \in K$, we have that $|s-z| \geq 1 / 2(2 n+1)$, for all $z \in Z\left(\psi_{F, k}\right)$. Then $K$ is uniformly bounded from the zeros of $\psi_{F, k}(s)$. Using Proposition 5 we see that (3.3) holds for $s \in K$ with $|m|$ sufficiently large. Therefore, in (3.2) we obtain that for $s=\sigma+i \tau$ with $0 \leq \sigma \leq \sigma_{0}$ and $\tau=T_{m}(|m|$ sufficiently large $)$ it follows

$$
F(s) \ll \frac{|\tau|^{\mu(\sigma)}}{|s-k-1|} .
$$

Using the fact that $\mu(\sigma) \leq 1$, we conclude that

$$
|F(s)| \ll 1<e^{|s|} .
$$

Now, we choose $T_{m}^{*}=-T_{-m}$, for all $m \in \mathbb{N}$. Thus, we obtain (ii) of Theorem 4 . Therefore

$$
N\left(-\sigma_{0}, \sigma_{0},-T, T, C_{F}\right)-N_{0}^{\prime}\left(T, C_{F}\right) \leq 4 \widehat{N}\left(0, \sigma_{0},-\varphi(2 T), \varphi(2 T), h\right)+O(1) .
$$


To conclude we need to bound $\widehat{N}\left(0, \sigma_{0},-\varphi(2 T), \varphi(2 T), h\right)$. Firstly, we choose $0<$ $\varepsilon<1 / 4$ such that $h(s)$ and $\psi_{F, k}(s)$ do not vanish on $\operatorname{Re} s=-\varepsilon_{0}$. The definition of $T_{m}$ implies that

$$
\frac{1}{2 n+1} \leq T_{m+1}-T_{m} \leq 2
$$

and using Proposition 5 we obtain $N\left(-\varepsilon, \sigma_{0}, T_{m}, T_{m+1}, \psi_{F, k}\right) \leq 2 n$. Let us divide the rectangle $\left\{s \in \mathbb{C}:-\varepsilon<\operatorname{Re} s<0\right.$ and $\left.T_{m}<\operatorname{Im} s<T_{m+1}\right\}$ into $2 n+1$ vertical subrectangles with horizontal length $\varepsilon /(2 n+1)$. So, one of these rectangles, denoted by $I_{m}$, has no zeros of $\psi_{F, k}(s)$ and $h(s)$. Suppose that the right vertical side of $I_{m}$ is contained on the line $\operatorname{Re} s=-\varepsilon_{m}$ that we can suppose without loss of generality that does not contain a zero of $\psi_{F, k}(s)$. Now, if we place a circle of radius $\delta>0$ sufficiently small (for instance $\delta<1 /(2 n+1)(16 n)$ ) we can enclosed the zeros of the rectangle $J_{m}=\left\{s \in \mathbb{C}:-\varepsilon_{m}<\operatorname{Re} s<\sigma_{0}\right.$ and $\left.T_{m}<\operatorname{Im} s<T_{m+1}\right\}$ in a contour $C_{m}$ such that the distance between $C_{m}$ and $J_{m}$ is at least $1 /(2 n+1)(16 n)$ and $C_{m}$ is distanced at least $1 /(2 n+1)(32 n)$ from the zeros of $\psi_{F, k}(s)$. We set $K=$ $\bigcup_{m} \partial J_{m} \quad\left(\partial J_{m}=\right.$ the boundaries of $\left.J_{m}\right)$. Then, applying (2.5) and Proposition 5 , we can find $M>0$ such that

$$
\left|b_{k} \psi_{F, k}(s)-\frac{h(s)}{\Gamma(s-k)}\right|<M<\left|b_{k} \psi_{F, k}(s)\right|
$$

for $s \in K$, with $|m|$ sufficiently large. If we denote $w(s)=h(s) / \Gamma(s-k)$, applying Rouché's theorem we obtain that there is $m_{0} \in \mathbb{N}$ sufficiently large such that

$$
N\left(-\varepsilon_{m}, \sigma_{0}, T_{m}, T_{m+1}, w\right)=N\left(-\varepsilon_{m}, \sigma_{0}, T_{m}, T_{m+1}, \psi_{F, k}\right),
$$

and

$$
N\left(-\varepsilon_{-m-1}, \sigma_{0}, T_{-m-1}, T_{-m}, w\right)=N\left(-\varepsilon_{-m-1}, \sigma_{0}, T_{-m-1}, T_{-m}, \psi_{F, k}\right)
$$

for $m \geq m_{0}$.

Finally, let $T$ be a positive real parameter. If $T<T_{m_{0}}$ we obtain $N\left(0, \sigma_{0}, 0, T, h\right)=$ $O$ (1). If $T \geq T_{m_{0}}$, we choose $m_{1} \geq m_{0} \geq 1$ such that $m_{1}<T_{m_{1}} \leq T<T_{m_{1}+1}<$ $m_{1}+2$. Since that the zeros of $1 / \Gamma(s)$ are the non-positive integers, by (3.6), Proposition 5 and (3.5), we get

$$
\begin{aligned}
\widehat{N}\left(0, \sigma_{0}, 0, T, h\right) & \leq \sum_{j=m_{0}}^{m_{1}} N\left(-\varepsilon_{j}, \sigma_{0}, T_{j}, T_{j+1}, h\right)+\widehat{N}\left(0, \sigma_{0}, 0, T_{m_{0}}+1, h\right) \\
& =\sum_{j=m_{0}}^{m_{1}} N\left(-\varepsilon_{j}, \sigma_{0}, T_{j}, T_{j+1}, w\right)+O(1)
\end{aligned}
$$




$$
\begin{aligned}
& =\sum_{j=m_{0}}^{m_{1}} N\left(-\varepsilon_{0}, \sigma_{0}, T_{j}, T_{j+1}, \psi_{F, k}\right)+O(1) \\
& \leq \sum_{j=m_{0}}^{m_{1}}\left(n+\frac{\ln (2 n+1)}{2 \pi}\left(T_{j+1}-T_{j}\right)\right)+O(1) \\
& \leq\left(n+\frac{\ln (2 n+1)}{\pi}\right) T+O(1) .
\end{aligned}
$$

Similarly, for $T<0$ we use (3.7) to obtain a similar bound. Thus, we obtain for $T>0$ that

$$
\widehat{N}\left(0, \sigma_{0},-T, T, h\right) \leq\left(2 n+\frac{2 \ln (2 n+1)}{\pi}\right) T+O(1) .
$$

We replace $T$ by $\varphi(2 T)$ in the above expression, and inserting in (3.4), and one can see that

$$
N\left(-\sigma_{0}, \sigma_{0},-T, T, C_{F}\right)-N_{0}^{\prime}\left(T, C_{F}\right) \leq\left(16 n+\frac{16 \ln (2 n+1)}{\pi}\right) T+O(1) .
$$

To obtain our desired result we will use an argument of $\mathrm{Ki}$ in [2, p. 131]. Following his idea, for $T>0$ we get that

$$
\begin{aligned}
& N\left(-\sigma_{0}, \sigma_{0},-T, T, C_{F}\right)-\overline{N_{1}}\left(T, C_{F}\right) \\
& \quad \leq 2\left(N\left(-\sigma_{0}, \sigma_{0}, 0, T, C_{F}\right)-\sum_{k=1}^{\infty} \overline{N_{k}}\left(T, C_{F}\right)\right),
\end{aligned}
$$

where $\overline{N_{k}}\left(T, C_{F}\right)$ denotes the number of zeros of $C_{F}$ with multiplicity $k$ with $|\operatorname{Im} s|<$ $T$ and $\operatorname{Re} s=0$, counting with multiplicity. Note that

$$
N_{0}^{\prime}\left(T, C_{F}\right) \leq \sum_{k=1}^{\infty} \overline{N_{k}}\left(T, C_{F}\right)
$$

We conclude combining (3.8), (3.9), (3.10), and recalling by (2.1) that $\bar{N}\left(T, C_{F}\right)=$ $N\left(-\sigma_{0}, \sigma_{0},-T, T, C_{F}\right)$.

\subsection{Proof of Theorem 3}

The proof is similar to the previous case. Using the function defined in (3.1), without loss of generality we can choose $\delta>0$ in such a way that $F(s) \neq \pm 1, h(s) \neq 0$ and $C_{F}(s) \neq 0$ when $\sigma=\delta$. By (2.5) and (2.6) it follows for $s=\sigma+i \tau$ with $0 \leq \sigma \leq \delta$ and $|\tau|$ sufficiently large 


$$
F(s)=\frac{O(1)|\tau|^{\mu(\sigma)}}{(s-k-1)\left(b_{k} \psi_{F, k}(s)+O\left(|s|^{-1 / 2}\right)\right)} .
$$

Using the fact that the $\psi_{F, k}(s)$ has no zeros in the strip $-\Delta_{* *}<\operatorname{Re} s<\Delta_{*}$, by Proposition 5 we get

$$
\left|b_{k} \psi(s-k)+O\left(|s|^{-1 / 2}\right)\right| \gg 1,
$$

for $s=\sigma+i \tau$, with $0 \leq \sigma \leq \delta$ and $|\tau|$ sufficiently large. Therefore

$$
|F(s)| \ll \frac{|\tau|^{\mu(\sigma)}}{|s-k-1|} .
$$

Using the fact that $\mu(\delta)<1$, then

$$
|F(s)| \ll \frac{|\tau|^{\mu(\delta)}}{|s-k-1|} \ll \frac{1}{|\tau|^{1-\mu(\delta)}}<1,
$$

for $s=\delta+i \tau$, with $|\tau|$ sufficiently large. Further, we have that $\mu(\sigma) \leq 1$, which implies in (3.12) that

$$
|F(s)| \ll \frac{|\tau|^{\mu(\sigma)}}{|s-k-1|} \ll 1<e^{|s|},
$$

for $s=\sigma+i \tau$ with $0 \leq \sigma \leq \delta$ and $|\tau|$ sufficiently large. Choosing $\varphi(x)=x+2$ and $T_{m}=T_{m}^{*}=m$, for $m$ sufficiently large, we get that the hypotheses in Theorem 4 are satisfied. Then

$$
N\left(-\delta, \delta,-T, T, C_{F}\right)-N_{0}^{\prime}\left(T, C_{F}\right) \leq 4 \widehat{N}(0, \delta,-\varphi(2 T), \varphi(2 T), h)+O(1) .
$$

Combining (2.5) and (3.11), we get a constant $L>0$ such that $|h(s)| \geq L|\Gamma(s-k)|$ for $s=\sigma+i \tau$ with $0 \leq \sigma \leq \delta$ and $|\tau|$ sufficiently large. Then, $h(s)$ only has finitely many zeros on the strip $0 \leq \sigma \leq \delta$, because all possible zeros are contained in a compact set. Therefore, the right-hand side in (3.13) is bounded and this implies our desired result.

Acknowledgements Part of the project was completed during my stay at IMCA with excellent working conditions.

Funding Open Access funding provided by NTNU Norwegian University of Science and Technology (incl St. Olavs Hospital - Trondheim University Hospital).

Open Access This article is licensed under a Creative Commons Attribution 4.0 International License, which permits use, sharing, adaptation, distribution and reproduction in any medium or format, as long as you give appropriate credit to the original author(s) and the source, provide a link to the Creative Commons licence, and indicate if changes were made. The images or other third party material in this article are included in the article's Creative Commons licence, unless indicated otherwise in a credit line to the material. If 
material is not included in the article's Creative Commons licence and your intended use is not permitted by statutory regulation or exceeds the permitted use, you will need to obtain permission directly from the copyright holder. To view a copy of this licence, visit http://creativecommons.org/licenses/by/4.0/.

\section{References}

1. Hardy, G.H.: Ramanujan: Twelve Lectures on Subjects Suggested by His Life and Work. Cambridge University Press, Cambridge (1940)

2. Ki, H.: On the zeros of approximations of the Ramanujan $\Xi$-function. Ramanujan J. 17(1), 123-143 (2008)

3. Levinson, N.: On theorems of Berlowitz and Berndt. J. Number Theory 3, 502-504 (1971)

4. Bellman, R., Cooke, K.L.: Differential-Difference Equations. Academic Press, New York (1963)

5. Levin, B.J.: Distribution of Zeros of Entire Functions. American Mathematical Society, Providence (1956)

6. de Bruijn, N.G.: The roots of trigonometric integrals. Duke Math. J. 17, 197-226 (1950)

7. Velásquez, O.: Majoration du nombre de zéros d'une fonction méromorphe en dehors d'une droite verticale et applications. J. Anal. Math. 110, 67-127 (2010)

Publisher's Note Springer Nature remains neutral with regard to jurisdictional claims in published maps and institutional affiliations. 